\title{
Role of intrauterine balloon stent or oral estrogen on prevention of adhesion after hysteroscopic septectomy in septate uterus: study protocol for a randomized controlled multi-center study in China
}

\section{Shan Deng}

Peking Union Medical College Hospital

\section{Zichen Zhao}

Peking Union Medical College Hospital https://orcid.org/0000-0002-8400-4484

\section{Limin Feng}

Beijing Tiantan Hospital

\section{Xiaowu Huang}

Beijing Fuxing Hospital

\section{Sumin Wang}

Nanjing Maternity and Child Health Care Hospital

\section{Xiang Xue}

The Second Affiliated Hospital of Xi'an Jiaotong University

\section{Lei Yan}

Reproductive Hospital Affiliated to Shandong University

\section{Baorong Ma}

Tianjin Central Hospital of Obstetrics and Gynecology

\section{Lijuan Hao}

Chongqing Health Center for Women and Children

\section{Xueying Li}

Hunan Province Maternal and Child Health Care Hospital

\section{Lihua Yang}

Tangshan Maternal and Child Health Care Hospital

\section{Liwen Song}

Tongren Hospital Shanghai Jiaotong University School of Medicine

\section{Heping Zhang}

Yale University School of Public Health

\section{Zijiang Chen}

Center for Reproductive Medicine, Cheeloo College of Medicine, Shandong University

\section{Lan Zhu ( $\square$ zhu_julie@vip.sina.com)}

Peking Union Medical College Hospital https://orcid.org/0000-0001-5753-5426 


\section{Study protocol}

Keywords: septate uterus, intrauterine adhesion, anti-adhesion agents, estrogen

Posted Date: April 13th, 2021

DOI: https://doi.org/10.21203/rs.3.rs-414374/v1

License: (c) (1) This work is licensed under a Creative Commons Attribution 4.0 International License. Read Full License 


\section{Abstract}

- Background

The septate uterus is the most common uterine malformation. The uterus septum increases the possibility of adverse pregnancy outcomes which can be improved by hysteroscopic metroplasty (transcervical resection of septate, TCRS). Various methods are used to prevent postoperative intrauterine adhesion in clinical practice. However, there is insufficient evidence to support or oppose the use of any of them. This study will be a multi-center, open labeled, prospective, randomized, controlled study. We will examine whether it is necessary to use the anti-adhesion barrier or a certain dose estrogen to prevent intrauterine adhesion after TCRS and further improve the fertility outcome.

- Methods

A total of 243 patients with septate uterus who meet the inclusion criteria will be randomly assigned into three groups with 1:1:1 allocation ratio. Group A will be the control group without any application of antiadhesion method after TCRS; the participants in group B will be inserted an intrauterine balloon stent after TCRS for one week; the participants in group C will take estradiol valerate ( $2 \mathrm{mg}$, bid) for 2 months after the surgery. A second-look hysteroscopy will be carried out after two menstrual cycles after the surgery to evaluate the intrauterine condition. Pregnancy outcomes, particularly the live birth rate, will be followed for at least one year.

- Discussion

This study will be the first well-powered clinical trial to assess the effectiveness of the anti-adhesion barrier or a certain dose of estrogen to prevent intrauterine adhesion after TCRS and further improve the fertility outcome. Our findings will guide the clinical care of patients after TCRS.

Trial registration: ChiCTR, ChiCTR2000032061. Registered on Apr 19, 2020, http://www.chictr.org.cn/showproj.aspx?proj=52499

\section{Introduction}

\section{Background and rationale $\{6 a\}$}

The septate uterus is the most common uterine anomaly, accounting for $35 \%$ - $90 \%$ of all identified uterine anomalies [1-4]. It is caused by abnormal resorption of the midline septum after the fusion of bilateral Mullerian ducts. According to ASRM-2016, the septate uterus is defined as the depth of internal fundal indentation $\geq 1.5 \mathrm{~cm}$ with acute angle of internal indentation ( $\nabla 90$ degree) [5]. The septum is variable in length and thickness, and may even extend into the cervix and vagina.

Septate uterus increases the risk of adverse reproductive and obstetric outcome. The patients with septate uterus have a higher risk of spontaneous abortion ( $21 \%-44 \%)$ and premature delivery $(12 \%-33 \%)$; 
and the live birth rate among these patients is $50 \%-72 \%[3,6,7]$. The pregnancy loss due to septate uterus usually occurs in the second trimester of pregnancy, which can be distinguished from the loss caused by cervical insufficiency according to the signs of labor [8]. Septate uterus can also increase the risk of breech presentation [8] and placental abruption [9]. The outcome of pregnancy can be improved by hysteroscopic septectomy (hysteroscopic metroplasty) $[5,8,10,11]$.

Hysteroscopic metroplasty is currently the first choice for patients with septate uterus. The greatest advantage of the TCRS is to maintain the integrity of uterine body without myometrial incision, that results in faster postoperative recovery, fewer complications and earlier to the subsequent pregnancy without interference with the choice of delivery mode.

There are different techniques and instruments for TRCS with the similarly high success rate and low complication rate. The application of microsurgical scissors or bipolar electrodes may improve surgical safety and reduce tissue damage compared with traditional unipolar instruments [12-14].

In addition to the usual ultrasonic monitoring, hysteroscopic metroplasty performed with the monitoring of laparoscopy can be used not only to evaluate the external outline of the uterus, but also to observe the integrity of the myometrium during the operation in order to reduce the risk of uterine perforation. The greatest advantage is that other pelvic reproductive disturbing factors can also be assessed simultaneously.

In view of the fact that all intrauterine procedures and operations may increase the risk of intrauterine adhesions, there are various anti-adhesion preparations and methods in clinical practice, such as intrauterine devices, Foley balloons, high-dose estrogen, and antibiotics [15]. However, there is insufficient evidence to support or oppose the use of any anti-adhesion agent or specific method (Level C). Previous studies suggest that endogenous estrogen is sufficient to stimulate the formation of endometrium within 2 months after hysteroscopic metroplasty [16]. However, the empirical uses of estrogen after surgery are quite common with no definite evidence.

\section{Objectives $\{7\}$}

To collect evidence that impacts clinical care, we design this trial to investigate whether it is essential to use the anti-adhesion barrier or a certain dose estrogen to prevent intrauterine adhesion after TRCS and improve the fertility outcome further.

\section{Trial design \{8\}}

This study is a multi-center, open labeled, prospective, randomized, controlled study. The participants will be randomly assigned into three groups with 1:1:1 allocation ratio. Superiority test will be used. 


\section{Methods: Participants, Interventions And Outcomes}

\section{Study setting $\{9\}$}

We will recruit 243 patients with septate uterus recruited from 12 grade-A tertiary hospitals in China (supplementary 1).

\section{Eligibility criteria $\{10\}$}

Inclusion criteria: women who are (1) $\leq 35$ years old; (2) nulliparous; (3) diagnosed with septate uterus;

(4) with regular menstrual cycles.

Exclusion criteria: women with (1) double cervix and/or vaginal septum; (2) intrauterine adhesions; (3) history of septectomy; (4) severe endometriosis and adenomyosis.

All the investigators of each center are member of hysteroscopy working group of minimally invasive technology committee of Chinese Obstetricians and Gynecologists Association. The study centres treat over 50\% of patients with septate uterus in China all together and all the surgeons are experienced with qualification of hysteroscopic surgery.

\section{Who will take informed consent? $\{26 \mathrm{a}\}$}

Patients admitted to the department of gynecology and obstetrics in each center are routinely screened for septate uterus. If the diagnosis is established for a patient, a study staff will screen her for eligibility and obtain study consent.

\section{Additional consent provisions for collection and use of participant data and biological specimens $\{26 \mathrm{~b}\}$}

Not applicable, no samples collected.

\section{Interventions}

\section{Explanation for the choice of comparators $\{6 \mathrm{~b}\}$}

No evidence supports a significant improvement of outcomes attributed to either of the two adhesion-preventive interventions we study. Therefore, the control group will receive the same surgery without any adhesionpreventive intervention.

\section{Intervention description $\{11 \mathrm{a}\}$}

A total of 243 patients with septate uterus who meet the inclusion criteria will be randomly assigned into three groups with 1:1:1 allocation ratio. Group A will be the control group without any application of anti-adhesion method after hysteroscopic metroplasty; the participants in group B will be placed with the intrauterine balloon stent during the operation for one week; the participants in group C will take estradiol valerate (2mg, bid) for 2 months after the surgery.

The participants will undergo hysteroscopic metroplasty within one week after the last menstrual bleeding. All the participants will be given a single dose of prophylactic antibiotics, commonly the secondgeneration cephalosporin, within 30 minutes before the surgery. If the patient is allergic to cephalosporin, other alternative drugs will be used as appropriate. The hysteroscopic metroplasty will be performed under laparoscopic monitoring. At the same time, the adhesion of fallopian tube and peritoneal endometriosis found by laparoscopy will be treated, and the polyps found by hysteroscopy will also be treated. Bipolar energy instruments (limited to electrical needles and electrical rings) will be used to incise or resect the uterine septum (and polyps) in each center. 


\section{Intrauterine balloon stent insertion}

For the participants in group B, the intrauterine balloon stent will be inserted into the uterine cavity during the operation. The stents used in different centers are all COOK balloon uterine stents $(5 \mathrm{~mL})$ without water injected into the balloon, and they will be taken out in the outpatient department one week later.

For the participants in group B, the intrauterine balloon stent will be inserted into the uterine cavity during the operation. The stents used in different centers are all COOK balloon uterine stents $(5 \mathrm{~mL})$ without water injected into the balloon, and they will be taken out in the outpatient department one week later.

\section{Administration of estrogen}

The participants in group $\mathrm{C}$ will start to take oral estradiol valerate (dose, $2 \mathrm{mg}$ bid) from the day after the surgery. If bleeding like menstruation occurs, the medication should be stopped for 5 days before being resumed. The management of bleeding during the medication will depend on the actual clinical condition of each patient. Progesterone can be added temporarily when there is no sign of ovulation.

\section{Hysteroscopic re-examination}

After surgery, the participants will undergo a hysteroscopic re-examination after two months. The hysteroscopy aims to evaluate the condition of uterine cavity. If a septum or intrauterine adhesion is observed, septum incision and adhesion lysis will be performed accordingly. If the residual septum is less than $0.5 \mathrm{~cm}$ in length, it will be only measured and recorded without further incision, because no evidence supports the influence of tiny residual septum on reproductive outcomes. If the residual septum is more than $0.5 \mathrm{~cm}$, additional excision will be performed. The location and texture of the adhesion should be recorded. COOK balloon or estrogen is not allowed to use during the re-examination surgery by any reason.

\section{Criteria for discontinuing or modifying allocated interventions $\{11 \mathrm{~b}\}$}

Both the theory and our former experience show that the dosage of the estrogen used in this trial is safe and may only cause slight adverse effects including unexpected bleeding which can be managed under the guidance from the researchers. Anaphylaxis is extremely rare. The complication of the COOK balloon stents is also rare and it will be taken out by the doctor in a week. Therefore, the discontinuing and dropping out is not easy to happen. But if the participant insists on withdrawing from the study, we will respect her choice.

\section{Strategies to improve adherence to interventions $\{11 \mathrm{c}\}$}

The participants will be fully informed of the trial before enrolment. For patients of group C, a couple of investigators are in charge of asking them the situation of medication using regularly. The researchers will answer the questions in 24 hours after the participants raise the question through the phone and give some guidance accordingly.

\section{Relevant concomitant care permitted or prohibited during the trial $\{11 \mathrm{~d}\}$}

The participants will be advised of strict contraception with non-hormone contraceptives before completing the second-look hysteroscopy. Progesterone can be used temporarily in the estrogen group for managing the bleeding issues.

\section{Provisions for post-trial care $\{30\}$}

All the participants will be followed up for more than 1 year after the second-look hysteroscopy. During the time, the researchers will provide free consultation on common obstetrical and gynecologic symptoms and make the diagnosis when necessary. The management-related medical costs will be at their own expense.

\section{Outcomes $\{12\}$}

The primary outcome is the rate of intrauterine adhesion confirmed by the second-look hysteroscopy. The secondary outcomes include live birth rate ( $>28$ weeks), clinical pregnancy rate, ongoing pregnancy rate ( $>20$ 
weeks), pregnancy loss rate, and operation-related complications.

\section{Participant timeline $\{13\}$}

The participant timeline is displayed in Table 1.

Table 1. Schedule of enrolment, interventions, and assessments.

\begin{tabular}{|c|c|c|c|c|c|c|}
\hline \multirow[b]{3}{*}{ TIMEPOINT } & \multicolumn{6}{|c|}{ STUDY PERIOD } \\
\hline & \multirow{2}{*}{$\begin{array}{c}\text { Enrolment } \\
\text { Day }-30 \\
\end{array}$} & \multirow{2}{*}{$\begin{array}{c}\text { Surgery } \\
\text { Day } 0\end{array}$} & \multicolumn{3}{|c|}{ Post-allocation } & \multirow{2}{*}{$\begin{array}{c}\text { Close-out } \\
\text { Pregnancy o } \\
\text { Month 15 } \\
\end{array}$} \\
\hline & & & Day 1 & Day 60 & etc. & \\
\hline \multicolumn{7}{|l|}{ ENROLMENT: } \\
\hline \multirow{2}{*}{$\begin{array}{l}\text { Eligibility screen } \\
\text { Informed consent }\end{array}$} & $x$ & & & & & \\
\hline & $\mathrm{X}$ & & & & & \\
\hline 3-D Ultrasound & $\mathrm{X}$ & & & & & \\
\hline \multicolumn{7}{|l|}{ BBT record } \\
\hline Allocation & & $x$ & & & & \\
\hline \multicolumn{7}{|l|}{ INTERVENTIONS: } \\
\hline \multicolumn{7}{|l|}{ No intervention } \\
\hline $\begin{array}{c}\text { Intrauterine stent } \\
\text { insertion }\end{array}$ & & $\mathrm{X}$ & & & & \\
\hline \multicolumn{7}{|l|}{ Estrogen } \\
\hline \multicolumn{7}{|l|}{ ASSESSMENTS: } \\
\hline $\begin{array}{l}\text { Hysteroscopic } \\
\text { re-examination }\end{array}$ & & & & $x$ & & $\mathrm{X}$ \\
\hline
\end{tabular}

\section{Baseline-day -30}

Baseline demographic information will be obtained from the participant's medical record: age, gravida, para, menstrual history, body mass index, and some other information. Three-dimensional ultrasound will be used to assess the uterine cavity in coronal position and measure the width and length of the septum. The informed consent will be obtained.

\section{Treatment-day 0}

The participants will be randomized into different groups on the day of the surgery. The hysteroscopic metroplasty will be performed uniformly under the monitoring of laparoscopy. During the operation, some other lesions (if any) will be treated at the same time, including fallopian tube adhesions, intraperitoneal endometriosis and endometrial polyps. At the end of the surgery, an intrauterine stent will be placed for the participants in group B.

\section{Post-operation-from day 1}

The participants in group $\mathrm{C}$ will start to take estradiol valerate from the day after the operation. Secondlook hysteroscopy will be performed two months later to avoid menstruation and observe the condition of the uterine cavity. If the uterine septum or intra-uterine adhesion is observed, further incision or 
adhesion separation will be carried out. If individual cases fail to make the second visit on schedule due to special reasons (such as the COVID-19 pandemic), the second-look hysteroscopy can be postponed, and the reasons need to be recorded clearly. The adverse event (AE) monitoring will be carried out weekly. The doctor will make a decision whether serious AEs necessitate the participant's withdrawal from the study.

\section{Follow-up}

Regular telephone follow-ups will be conducted every 3 months after the surgery until the pregnancy outcome occurs or the observational period ends, which is 24 months after the second-look hysteroscopy. Besides, we will establish a platform for patients' self-reporting. The information collected during the follow-up will be specified by the research team before the study.

\section{Sample size $\{14\}$}

According to the incidence of postoperative intrauterine adhesion reported in previous literature (ranging from $7 \%$ to $37.5 \%$ )[17-19], we selected $24 \%$ and $5 \%$ as the postoperative uterine adhesion rates respectively in the control group and the two intervention groups. Superiority test will be used. With the allocation ratio of the three groups being 1:1:1 and considering $\alpha=0.05$ and $\beta=0.20$, each group requires a sample size of 73 patients. Assuming 10\% attrition, 81 patients per group would be required, for a total of 243 patients. [20]

\section{Recruitment $\{15\}$}

The design of multi-centre is indented for more and faster enrolment of the participants. The patients meeting the inclusion criteria after screening will be informed by the senior doctors at each centre, thus promoting the patients' trust and their willingness in joining in the trial. Due to the COVID-19 pandemic, we will consider prolonging the duration of recruitment.

\section{Assignment of interventions: allocation}

\section{Sequence generation $\{16 \mathrm{a}\}$}

Randomization will be performed by a central randomization system on Clinical Trial Management Public Platform.[21] Each centre has an account and is distributed a specific number of participants to recruit. The information of the participants will be managed on this platform.

\section{Implementation $\{16 \mathrm{c}\}$}

The investigators at each centre are responsible for screening and enrolling. After obtaining informed consent, the information of the participants will be added into the Clinical Trial Management Public Platform and randomization will be performed with the use of the website. The qualified and experienced surgeons at each centre will conduct the surgery and insert the balloon stent.

\section{Assignment of interventions: Blinding}

\section{Who will be blinded $\{17 \mathrm{a}\}$}

After assignment, the investigators and the participants will know the specific intervention, but the outcome assessors and data analysts will be all blinded. The assignment will be presented as the symbol or coding number, concealing the exact intervention. 
After randomization, both the investigators and the participants will be informed of the allocation.

\section{Data collection and management}

\section{Plans for assessment and collection of outcomes $\{18 \mathrm{a}\}$}

The study team provides a unified Case Report Form (CRF) (supplementary 2) and a web-based data entry system to facilitate the data collection and track the enrolment status regularly. We cooperate with a third-party data management company to customize a mobile application for data inputting. The participants' information added by each center will be fed back to the organizer regularly by the data platform as required.

\section{Plans to promote participant retention and complete follow-up $\{18 \mathrm{~b}\}$}

To maximize data collection at 12 months after the second-look hysteroscopy, participants will be informed at discharge that they will be contacted every 3 months in the following year, and told the approximate date of this contact. If a participant fails to respond to us at 3-month contact, study staff will make every effort to regain contact (via telephone, email and, if necessary, a letter to their last known mailing address). These contact attempts will be documented. Should the participant continue to be unreachable, she will be considered to be lost to follow-up. These participants will not be withdrawn from the study, and existing data will be analyzed. Any incidents of non-retention and the reason for this will be documented.

\section{Data management $\{19\}$}

The management of patients' medical records complies with related regulations. Participant's case report forms and source documents will be stored securely at Peking Union Medical College Hospital (PUMCH). Data will be entered electronically from original study materials into a password-protected file for analysis. The data transmitted electronically will be password protected.

\section{Confidentiality $\{27\}$}

A dedicated staff takes charge of the paper and electronic materials involved in this study, and the access to the system is limited to protect the confidentiality of the information.

\section{Plans for collection, laboratory evaluation and storage of biological specimens for genetic or molecular analysis in this trial/future use $\{33\}$ \\ Not applicable, no samples collected.}

\section{Statistical methods}

\section{Statistical methods for primary and secondary outcomes $\{20 \mathrm{a}\}$}

Chi-square test will be used to compare the rates of three independent samples. P values of less than 0.05 are considered to indicate statistical significance. All the statistics analysis will be performed using SPSS software, version 22.0 . 
The interim analyses will be performed when one-third or half of the participants finish the second-look hysteroscopy. In order to avoid taking the bias caused by the interim results into the following research, the specialized statisticians free of clinical work will do the interim analyses. The interventions we use in this research are widely applied in clinical practice. Therefore, the possibility of pre-termination is slight.

\section{Methods for additional analyses (e.g. subgroup analyses) $\{20 \mathrm{~b}\}$}

We will further use multinomial logistic regression to adjust for the potential effect of age, type or length of septum and some other baseline characteristics indicators on the outcomes of interest.

\section{Methods in analysis to handle protocol non-adherence and any statistical methods to handle missing data $\{20 \mathrm{c}\}$}

We will use listwise deletion to handle missing values.

\section{Plans to give access to the full protocol, participant level-data and statistical code \{31c\}} Patients' records are anonymized to protect privacy. Full protocol will be disseminated through peer-reviewed publications. Statistical code is not applicable in our research.

\section{Oversight and monitoring}

\section{Composition of the coordinating centre and trial steering committee $\{5 \mathrm{~d}\}$}

The trial is performed under the guidance and monitoring of the steering committee. It will be overseen by the protocol committee and the data coordination committee, consisted by investigators from different centers. The principal investigator will report the progress of the trial to the Institutional Review Board (IRB) of Peking Union Medical College Hospital (PUMCH) once a year.

\section{Adverse event reporting and harms $\{22\}$}

All AEs and abnormal laboratory values will be recorded in the subjects' case reporting form. Each AE will be followed until it is resolved, stabilized or until the follow-up period ends. The AEs that may be associated with the study intervention(s) will be followed until the event (or its sequelae) is resolved or stabilizes at an acceptable level to the primary physician and patient. All the serious AEs need to be reported in the timeframe required by Ethic Committee and local authorizations. Site PI is required to report the serious AE timely by filling in the Serious Adverse Event Report Form within 24 hours of discovery.

\section{Frequency and plans for auditing trial conduct $\{23\}$}

The interim quality control meeting will be held every 6 months to monitor the research progress of each center, and the data are not disclosed in order to prevent the bias. The registration forms of screened cases and the enrolled participants are required to submit monthly. The electronic information form is required to be filled within 1 week after each operation. Each center will perform spot checks of electronic medical records regularly or irregularly, and conduct on-site supervision when necessary.

The investigators will allow study-related monitoring, including institutional ethics committee's review and regulatory inspection(s), and provide access to relevant data or documents. 


\section{Plans for communicating important protocol amendments to relevant parties (e.g. trial participants, ethical committees) $\{25\}$}

Any protocol modification, including changes to eligibility criteria, outcomes and analyses will be reported to the ethical committees in time and informed the investigators at each centre through telephone, email and WeChat group. If the amendment concerns the participants, the investigators at each centre will be in charge of communicating with the participants enrolled there.

\section{Dissemination plans $\{31 \mathrm{a}\}$}

The results of this study will be published in international peer-reviewed journals and presented at relevant research conferences. We expect to submit the primary research paper within 6 months after the study ends.

\section{Discussion}

Hysteroscopic metroplasty is the management for most patients with septate uterus and many patients develop with intrauterine adhesions after the surgery. However, there is a lack of evidence on the efficacy of intra- or post-operative anti-adhesion interventions. This trial aims to examine the effect of intrauterine balloon stent and estrogen on reproductive outcome after hysteroscopic metroplasty.

This study also has important strengths. It is rigorously designed with adequate power. Despite the recruitment challenge noted above, the large population in China ensures a large pool of potential patients, and hence we expect to overcome the challenge and complete the recruitment in a relatively short time. The results of this trial will provide high-quality evidence to guide the clinical practice on adhesion prevention, which can prevent unnecessary use of drugs and appliances.

This trial has some potential limitations and challenges. The recruitment is anticipated as a major challenge in this trial. Although the septate uterus is the most common anomaly of the Mullerian ducts, the incidence is relatively low, and it is not frequently screened among the women without reproductive problems. The screening criteria in this trial also increases the difficulty of recruitment by excluding the patients with cervical or vaginal septum, and the comorbidities affecting the reproductive outcome including severe adenomyosis, severe endometriosis and intrauterine adhesion. The multi-center design can help recruit more patients. To ensure data quality, uniform training and regular monitoring are essential in the implementation of this trial. One possible drawback of this trial is that monitoring by laparoscopy may cause extra pain to the participants, thus making the informed consent more difficult to obtain. Finally, this study only tests one specific balloon stents (COOK) and the estrogen is applied in only one dose of 4 milligrams per day, without comparison by other appliances or dosages.

\section{Trial Status}


Recruitment for this project began in May 2020 and is expected to be completed by March 2022. The current recruitment status is 100 . The protocol version at the time of submission is version 1.0 (Jan. 1st, 2019).

\section{Abbreviations}

$\mathrm{AE}$

Adverse event; CAMS:Chinese Academy of Medical Sciences; CRF:Case Report Form; COVID-19:Corona Virus Disease 2019; PI:Principal investigator; PUMCH:Peking Union Medical College Hospital; SPSS:Statistical Product and Service Solutions; TCRS:Trans-cervical resection of septate

\section{Declarations}

\section{Acknowledgements}

We would like to thank the person to contact at each centre (Jinghua Li, Haiyan Wang, Xiangdong Hua, Ruonan Xu, Yousheng Zhang, Danfeng Gao, Yun Yang, Li Chen, Shasha Rao, Qiuhong Liu, Yaling Sun) for their contribution in implementation of this trial. We would like to thank Weijie Tian for his help in registering and applying working account. We would like to thank Haiqing Zhan and Jing Dong for their work in development and maintenance of the data input platform.

\section{Authors' contributions $\{31 \mathrm{~b}\}$}

LZ and ZC conceived and designed the study. SD registered this study. SD and ZZ drafted this manuscript. All the authors reviewed and edited the protocol. All the authors read and approved the manuscript. The data management platform will generate the allocation sequence, the PI of each centre will enroll participants, and will assign participants to interventions. Each centre will collect their participants' medical records and the researchers in PUMCH will collect all the data.

\section{Funding $\{4\}$}

This project is funded by Chinese Academy of Medical Sciences (CAMS) Initiative for Innovative Medicine (CAMS-I2M; 2017-I2M-1-002).

Study design, data collection, management, analyses, interpretation of data and report writing and submission are entirely independent of the study sponsor and sources of financial and other support. 
The Pls at each centre will have access to the final trial dataset. Data transmitted electronically will be password protected.

\section{Ethics approval and consent to participate $\{24\}$}

This protocol and other relevant materials were approved by the Ethics Committee of Peking Union Medical College Hospital. Ethics approval will be obtained from each participating center. This study is compliant with the clinical guidelines and regulations for septate uterus treatment, and will not increase the risks of patients. This protocol is designed and approved by all the investigators from each study site. Any modification to the protocol will require a formal protocol amendment. All the principal investigators will be well-trained. Written, informed consent will be obtained prior to the patient's enrolment into the trial.

\section{Consent for publication $\{32\}$}

Not applicable.

\section{Competing interests $\{28\}$}

The authors declare that they have no competing interests.

\section{References}

1. Simón C, Martinez L, Pardo F, et al. Müllerian defects in women with normal reproductive outcome. Fertil Steril. 1991;56:1192.

2. Acién P. Incidence of Müllerian defects in fertile and infertile women. Hum Reprod. 1997;12:1372.

3. Grimbizis GF, Camus M, Tarlatzis BC, et al. Clinical implications of uterine malformations and hysteroscopic treatment results. Hum Reprod Update. 2001;7:161.

4. Rikken JF, Kowalik CR, Emanuel MH, et al. Septum resection for women of reproductive age with a septate uterus. Cochrane Database Syst Rev. 2017;1:CD008576.

5. Practice Committee of the American Society for Reproductive Medicine. Electronic address: ASRM@asrm.org, Practice Committee of the American Society for Reproductive Medicine. Uterine septum: a guideline. Fertil Steril. 2016;106:530.

6. Ludmir J, Samuels P, Brooks S, Mennuti MT. Pregnancy outcome of patients with uncorrected uterine anomalies managed in a high-risk obstetric setting. Obstet Gynecol. 1990;75:906.

7. Heinonen PK. Complete septate uterus with longitudinal vaginal septum. Fertil Steril. 2006;85:700. 
8. Valle RF, Ekpo GE. Hysteroscopic metroplasty for the septate uterus: review and meta-analysis. J Minim Invasive Gynecol. 2013;20:22.

9. Venetis CA, Papadopoulos SP, Campo R, et al. Clinical implications of congenital uterine anomalies: a meta-analysis of comparative studies. Reprod Biomed Online. 2014;29:665.

10. Fedele L, Bianchi S. Hysteroscopic metroplasty for septate uterus. Obstet Gynecol Clin North Am. 1995;22:473.

11. Fedele L, Bianchi S. Hysteroscopic metroplasty for septate uterus. Obstet Gynecol Clin North Am. 1995;22:473.

12. Candiani GB, Vercellini P, Fedele L, et al. Argon laser versus microscissors for hysteroscopic incision of uterine septa. Am J Obstet Gynecol. 1991;164:87.

13. Vercellini P, Vendola N, Colombo A, et al. Hysteroscopic metroplasty with resectoscope or microscissors for the correction of septate uterus. Surg Gynecol Obstet. 1993;176:439.

14. Colacurci N, De Franciscis P, Mollo A, et al. Small-diameter hysteroscopy with Versapoint versus resectoscopy with a unipolar knife for the treatment of septate uterus: a prospective randomized study. J Minim Invasive Gynecol. 2007;14:622.

15. Vercellini P, Fedele L, Arcaini $L$, et al. Value of intrauterine device insertion and estrogen administration after hysteroscopic metroplasty. J Reprod Med. 1989;34:447.

16. Candiani GB, Vercellini P, Fedele L, et al. Repair of the uterine cavity after hysteroscopic septal incision. Fertil Steril. 1990;54:991.

17. Taskin 0 , Sadik S, Onoglu A, et al. Role of endometrial suppression on the frequency of intrauterine adhesions after resectoscopic surgery. J Am Assoc Gynecol Laparosc. 2000;7(3):351-4.

18. Yu X, Yuhan L, Dongmei $S$, et al. The incidence of postoperative adhesion following transection of uterine septum: a cohort study comparing three different adjuvant therapies. Eur J Obstet Gynecol Reprod Biol. 2016;201:61-4.

19. Salazar CA, Isaacson K, Morris S. A comprehensive review of Asherman's syndrome: causes, symptoms and treatment options. Curr Opin Obstet Gynecol. 2017;29(4):249-56.

20. Power and Sample Size. http://powerandsamplesize.com. Accessed Jan. 1st, 2019.

21. Clinical Trial Management Public Platform. http://www.medresman.org. Accessed Jan. 1st, 2019.

\section{Supplementary Files}

This is a list of supplementary files associated with this preprint. Click to download.

- Supplementary1Listofstudysites.docx

- Supplementary2CRF.doc 